\title{
Exploratory analysis of transposable elements expression in the C. elegans early embryo
}

Federico Ansaloni ${ }^{1}$, Margherita Scarpato ${ }^{2}$, Elia Di Schiavi ${ }^{3}$, Stefano Gustincich ${ }^{1,2^{*}}$ and Remo Sanges ${ }^{1,4^{*}}$ (D)

From Annual Meeting of the Bioinformatics Italian Society (BITS 2018)

Turin, Italy. 27 - 29 June 2018

\begin{abstract}
Background: Transposable Elements (TE) are mobile sequences that make up large portions of eukaryote genomes. The functions they play within the complex cellular architecture are still not clearly understood, but it is becoming evident that TE have a role in several physiological and pathological processes. In particular, it has been shown that TE transcription is necessary for the correct development of mice embryos and that their expression is able to finely modulate transcription of coding and non-coding genes. Moreover, their activity in the central nervous system (CNS) and other tissues has been correlated with the creation of somatic mosaicisms and with pathologies such as neurodevelopmental and neurodegenerative diseases as well as cancers.

Results: We analyzed TE expression among different cell types of the Caenorhabditis elegans (C. elegans) early embryo asking if, where and when TE are expressed and whether their expression is correlated with genes playing a role in early embryo development. To answer these questions, we took advantage of a public C. elegans embryonic single-cell RNA-seq (sc-RNAseq) dataset and developed a bioinformatics pipeline able to quantify reads mapping specifically against TE, avoiding counting reads mapping on TE fragments embedded in coding/non-coding transcripts. Our results suggest that i) canonical TE expression analysis tools, which do not discard reads mapping on TE fragments embedded in annotated transcripts, may over-estimate TE expression levels, ii) Long Terminal Repeats (LTR) elements are mostly expressed in undifferentiated cells and might play a role in pluripotency maintenance and activation of the innate immune response, iii) non-LTR are expressed in differentiated cells, in particular in neurons and nervous system-associated tissues, and iv) DNA TE are homogenously expressed throughout the C. elegans early embryo development.

Conclusions: TE expression appears finely modulated in the C. elegans early embryo and different TE classes are expressed in different cell types and stages, suggesting that TE might play diverse functions during early embryo development.
\end{abstract}

Keywords: Transposable elements, Caenorhabditis elegans, Early embryo, Embryogenesis, RNA-Seq, Single-cell, Bioinformatics

\footnotetext{
* Correspondence: Stefano.Gustincich@iit.it; remo.sanges@gmail.com

${ }^{1}$ Area of Neuroscience, International School for Advanced Studies (SISSA),

Trieste, Italy

Full list of author information is available at the end of the article
}

(c) The Author(s). 2019 Open Access This article is distributed under the terms of the Creative Commons Attribution 4.0 International License (http://creativecommons.org/licenses/by/4.0/), which permits unrestricted use, distribution, and reproduction in any medium, provided you give appropriate credit to the original author(s) and the source, provide a link to the Creative Commons license, and indicate if changes were made. The Creative Commons Public Domain Dedication waiver (http://creativecommons.org/publicdomain/zero/1.0/) applies to the data made available in this article, unless otherwise stated. 


\section{Background}

Transposable Elements (TE) are repetitive elements spread among the genomes of almost all eukaryotes [1]. TE can be classified in transposons and retrotransposons according to their mechanism of transposition. Transposons are composed by DNA and rolling-circle (RC) elements and mobilize through a DNA intermediate, while retrotransposons are composed by Long Terminal Repeats (LTR) and non-LTR (LINE and SINE) sequences that take advantage of an mRNA intermediate for their mobilization $[1,2]$. TE make up a large portion of human and murine genomes (40-45\%) and despite having been understudied and often considered as junk and selfish elements, it is currently believed that they have played and continue to play important roles in the biology and evolution of metazoan [2-6]. One of the first observation of the existence and activity of TE was made in Drosophila melanogaster where specific outcrosses displayed sterility and other germline abnormalities defined together as hybrid dysgenesis. Further observations lead to the discovery that these phenotypes were due to the lack of silencing, in the specific outcrosses, of the Pelement (a DNA transposon) and elucidated the molecular mechanisms causing the phenomenon [7]. Later, Mello and Fire discovered that Caenorhabditis elegans (C. elegans) mutants, deficient for RNA interference (RNAi), displayed an increased TE mobilization and proposed that the RNAi system has evolved also as a defence response to protect germline from TE activity [8]. Nowadays, although TE activity in the gonads might represent a driving force in genome evolution, it is accepted that it is mostly inhibited by the PIWI/piRNA pathway [9]. Findings in the last decade highlighted that TE mobilization is not confined to germ cells and cancer tissues. They, indeed result expressed and active during embryogenesis [10-17] and even in the adult central nervous system (CNS) [5, 18-23]. TE (mostly LTR) have been proposed to play fundamental roles during embryogenesis, when they shape gene expression acting as regulatory elements, providing promoters and binding sites, regulating chromatin accessibility, and physically interacting with transcripts $[10,11]$. Several studies evidenced that TE are needed during mammalian embryogenesis in diverse biological processes such as pluripotency maintenance, embryo viability and immune response priming [12-15]. According to these studies, the complete lack of expression as well as the over-expression of TE is not compatible with the correct development of the mammalian embryo, thus suggesting that the expression of TE is strictly regulated during mammalian embryogenesis. Finally, TE have been suggested to play a dual role in the CNS of organisms such as fruitfly, mouse and human. On one hand, activity of retrotransposons in CNS determines somatic mosaicism [5, 18-23] which has been proposed to be correlated with the evolution of cognitive capabilities $[19,20,22]$. On the other, alteration of their expression and activity have been associated to neurodevelopmental and neurodegenerative disorders [24-27].

C. elegans is a $\sim 1 \mathrm{~mm}$ long nematode largely used as model organism. Its maintenance under laboratory conditions is very simple: the transparent nematode is characterized by a short generation time (3-4 days), its food source is Escherichia coli and up to 1000 worms can be cultured at the same time in a $55 \mathrm{~mm}$ petri dish [28]. $C$. elegans embryogenesis lasts for $\sim 16 \mathrm{~h}$, the embryonic cell lineage has been the first metazoan to be completely mapped in the early eighties and a name has been assigned to all the embryonic cells [29]. In the early stages five asymmetric divisions produce six founder cells: AB, MS, E, C, D, and P4. In more details a P0 zygote gives rise to a larger anterior cell, $\mathrm{AB}$, and a smaller posterior blastomere, P1 (2-cell stage). P1 undergoes an asymmetric division that gives rise to EMS and P2, while $\mathrm{AB}$ through a symmetric division gives raise to $\mathrm{ABa}$ and ABp (4-cell stage). Subsequent asymmetric divisions of EMS into MS and E, of P2 into C and P3, and symmetric divisions of $\mathrm{ABa}$ and $\mathrm{ABp}$, which generate $\mathrm{ABal}, \mathrm{ABar}$, $\mathrm{ABpl}$ and $\mathrm{ABpr}$, characterize the 8-cell stage. The further divisions of the 8 cells complete the generation of the founder cells whose descendants will produce specific cell types (16-cell stage) [29]. C. elegans gene manipulation can be carried out in simple and very effective ways $[30,31]$. The adult is composed of about 1000 somatic cells, 302 of which are neurons. Approximately 15\% of its genome derives from TE [32]. Unlike fruitfly, mouse and human genomes in which the majority of TE are retrotransposons, C. elegans TE are mostly DNA transposons (Additional file 1). Globally, $74 \%$ of C. elegans TE are annotated as DNA transposons, $16 \%$ as RC transposons and $10 \%$ as retrotransposons (1\% SINE, $4 \%$ LINE, 5\% LTR). According to literature, the Tc/Mar family (DNA TEs) is the most active, while active retrotransposition was never observed under laboratory conditions [32, 33]. To our knowledge no study has ever been performed on the expression of TE during C. elegans development.

Here we explore TE expression dynamics in the C. elegans early embryo (from the zygote to the 16-cell stage) taking advantage of the single-cell RNA sequencing (scRNA-seq) dataset generated by Tintori et al. in 2016 [34]. We developed a bioinformatics pipeline aimed at the quantification of TE-specific reads and analyzed if, when and where each specific class of TE is expressed during $C$. elegans development and their potential correlations with the expression of protein coding genes.

\section{Methods}

Data collection and pre-processing

To study the expression of TE in the C. elegans early embryo we took advantage of Tintori et al. scRNA-seq public 
data [34]. They sequenced single cells from embryos of the 1-, 2-, 4-, 8- and 16-cell stage. Totally, they sequenced 219 cells, generating between 5 and 9 replicates for each of the 31 different cell types. We downloaded raw files containing single-end reads of $50 \mathrm{bp}$ from ENA-EBI database (PRJNA312176 accession code) and discarded 55 samples that did not pass quality filters regarding whole embryo mRNA mass, according to the authors. The filtered dataset is globally composed of 164 samples, each cell type is represented by a minimum of 4 to a maximum of 7 replicates. We report the selected samples in Additional file 2.

\section{TE expression analysis}

We downloaded the fasta files containing coding (cDNA file - WB235 version) and non-coding transcripts (ncRNA file - WB235 version) from Ensembl (version 93) [35] and the fasta file containing the nucleotidic sequences of all the C. elegans repeats from RepBase database [36] which we call the transposome. We discarded from the transposome 16 sequences annotated as Satellite (SAT). We then developed a bioinformatics pipeline (a brief workflow of pipeline rationale is represented in Fig. 1a, b) able to select reads mapping specifically on transposome and not on TE fragments embedded in coding and/or long non-coding transcripts. We used STAR [37] (version 2.6.0c, parameters: --outSAMprimaryFlag AllBestScore, --outFilterMismatchNoverLmax 0.04) to map the reads on the reference obtained merging the described RepBase transposome and the Ensembl transcriptome files containing coding and non-coding transcripts, assigning primary alignment flag to all the alignments with the best score. Using samtools [38] (v1.3.1) we selected alignments flagged as primary $(-\mathrm{F} 0 \times$ 100 parameter). To avoid selection of reads mapping on TE fragments embedded in coding and/or long non-coding transcripts we discarded reads mapping with best-scoring alignments both on transposome and transcriptome using custom Python scripts and Picard FilterSamReads tool [39]. Using bedtools coverage [40] (v2.26.0, -counts parameter) we counted for each sample the number of selected reads mapping exclusively on TE. Raw counts have then been normalized on the total number of mapping reads and multiplied by 1000,000 obtaining expression values indicated as reads per million mapped reads (RPM). To test the accuracy of our pipeline we carried out the same analysis using the recent published SalmonTE pipeline [41] that measures TE expression levels using the Salmon tool on RNA-seq data and a set of transposon consensus sequences. First, using the SalmonTE index mode (--te_only parameter) we created the index file starting from the $C$. elegans consensus sequences fasta file downloaded from RebBase database. Then, taking advantage of the SalmonTE quant mode (--exprtype = count parameter) we quantified the TE expression values. Finally, we selected the TE in common between the two analyses and made the comparison between the tools. To perform the principal component analysis (PCA) represented in Fig. 2b we first selected DNA, LTR, LINE and SINE TE and then grouped together the expression values of elements belonging to the same class. The expression values where then transformed using a square root transformation and the PCA analysis was executed using the fast.prcomp() function of the gmodels R library. The first two components were plotted using the ggbiplot() function of the ggbiplot R library.

\section{$\mathrm{TE} /$ gene expression correlation and pathways analysis} We performed a correlation analysis between the expression of the analyzed TE and all the C. elegans genes. We retrieved gene expression values (RPKM) from the Supplementary Table S2 of the paper published by Tintori et al. [34]. To select TE and genes with a reproducible expression among the replicates of the same cell type we selected TE and mRNAs with an expression value $>=25$ RPM or RPKM in at least 3 replicates of at least 1 cell type. We performed a pairwise correlation analysis between TE and coding genes using Pearson correlation test. Pearson correlation coefficients were calculated using pearsonr function of the scipy Python module (stats sub-module) selecting only correlations with $R^{2}>=0.4$ or $<=-0.4$ and with an FDR (Benjamini \& Hochberg) corrected $p$-value $<=0.0001$. To analyze potential pathway enrichment for genes involved in the selected correlations, a statistical over-representation test was performed using Panther tool [42] (version: 13.1, reference list: Caenorhabditis elegans, Annotation Data Set: Reactome pathways, Test type: Fisher's Exact with FDR multiple test correction, FDR corrected p-value cut-off $<0.01)$. All the plots were generated using $R$ Software.

\section{Results and discussion \\ A bioinformatics pipeline to specifically measure TE expression levels}

Taking advantage of the scRNA-seq dataset published by Tintori et al [34], we quantified TE expression in all the sampled cells. This dataset is composed of 164 samples subdivided among the 31 different cell types characterizing 5 early embryo cell stages (1-, 2-, 4-, 8- and 16-cell stages). To consider only reads effectively mapping on TE, our pipeline specifically exclude reads mapping on TE fragments embedded in annotated coding and/or long non-coding transcripts. Reads are firstly mapped, allowing multimapping, against a reference transcriptome made of all the annotated transcripts plus the entire species-specific TE consensus sequences from RepBase. Next, for each read, all the alignments with the best score (multimapping reads may have more than one alignment with best score) are selected. Finally, reads aligning with best score exclusively against TE 


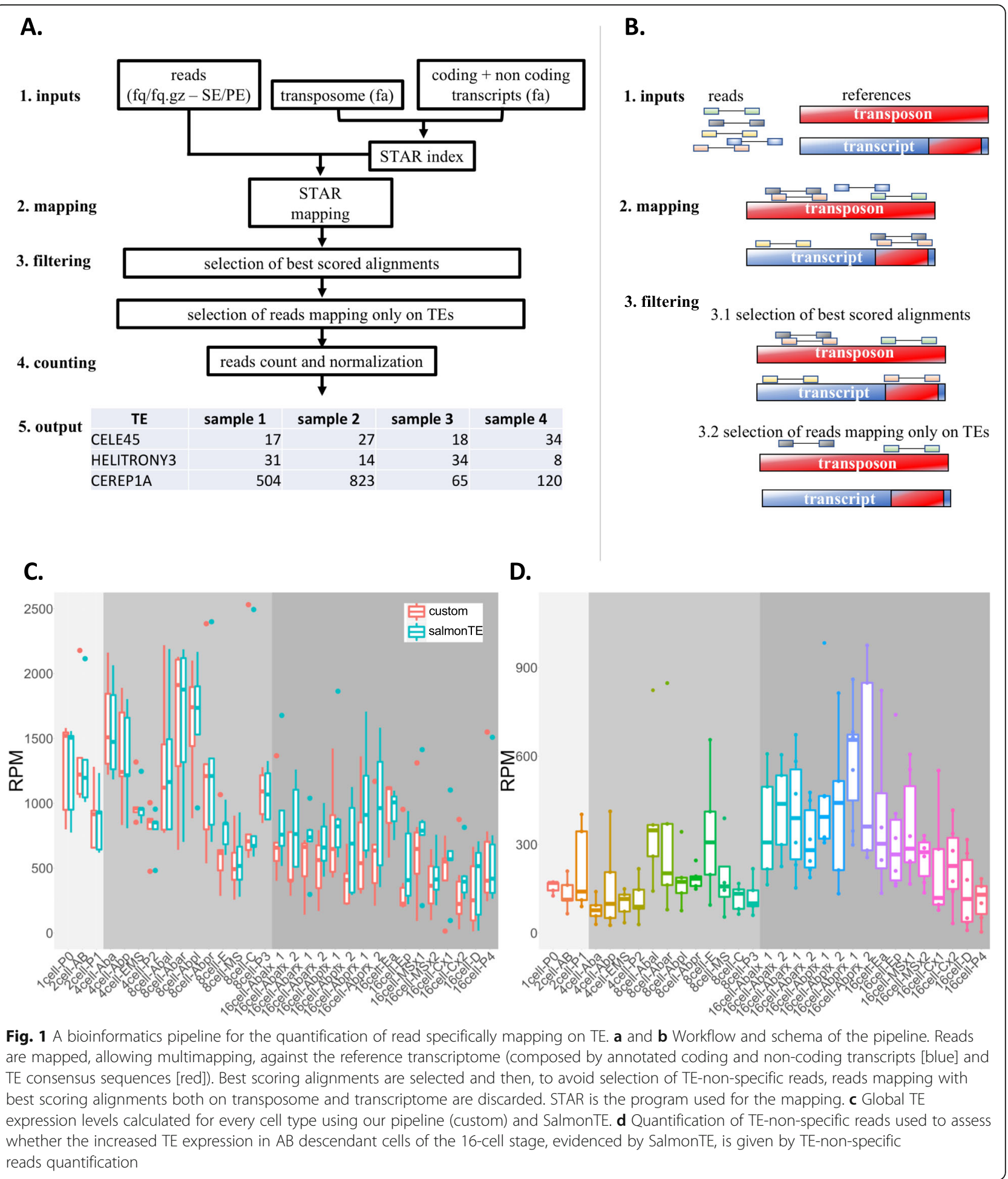

are used for TE expression quantification. This means that a read mapping with the best score against both a TE and a coding/non-coding transcript is discarded (Fig. 1a, b). This strategy avoids the usage of those reads that might derive from TE fragments embedded in annotated transcripts in the measurement of TE expression. In this work, we will call
TE-non-specific the reads mapping with best alignment score on both a TE and a coding/non-coding transcript and $T E$-specific those reads mapping with the best score exclusively on a TE. On average, about $80 \%$ of reads were mapped against the whole reference transcriptome (the union of coding, non-coding and TE transcripts). TE 


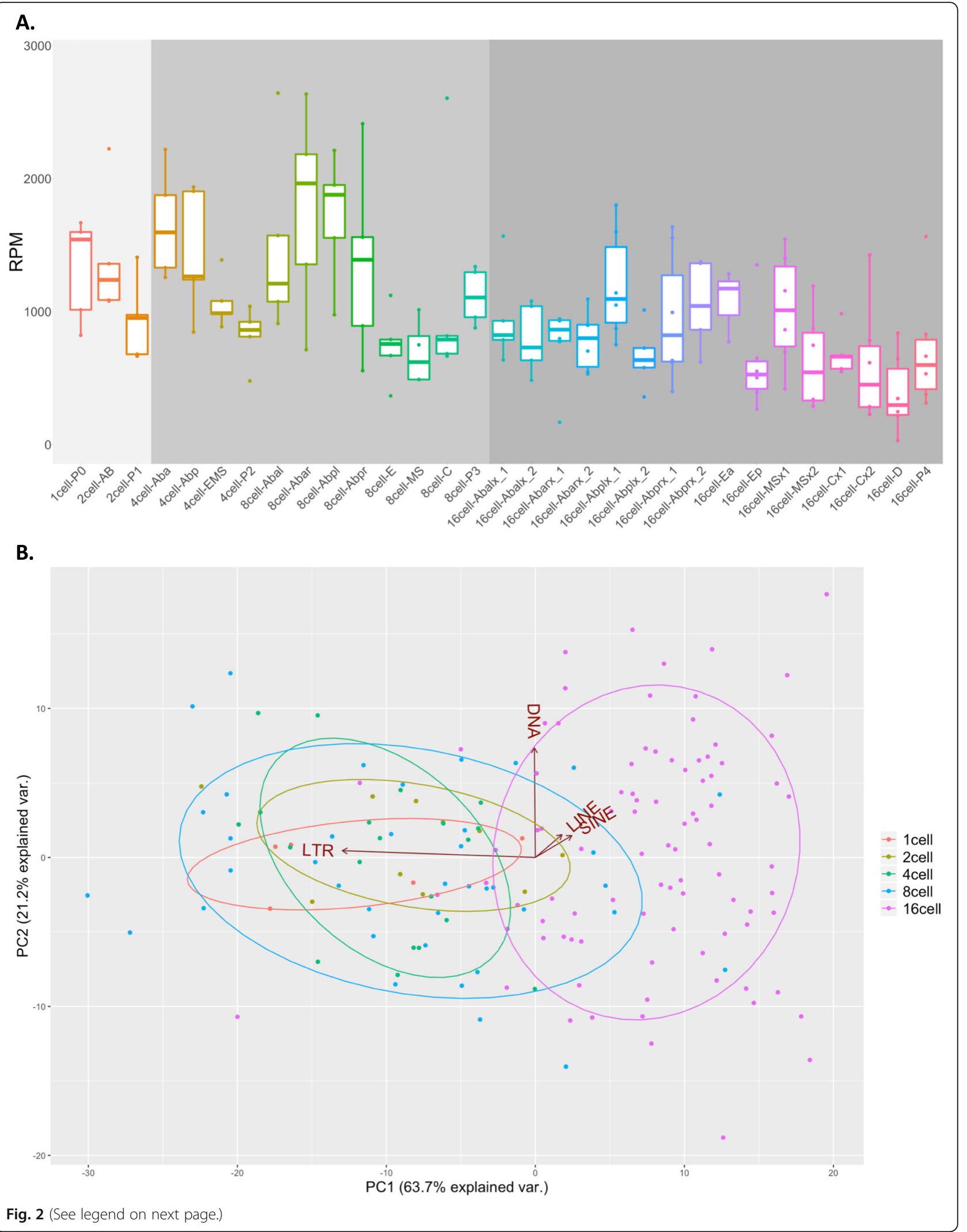




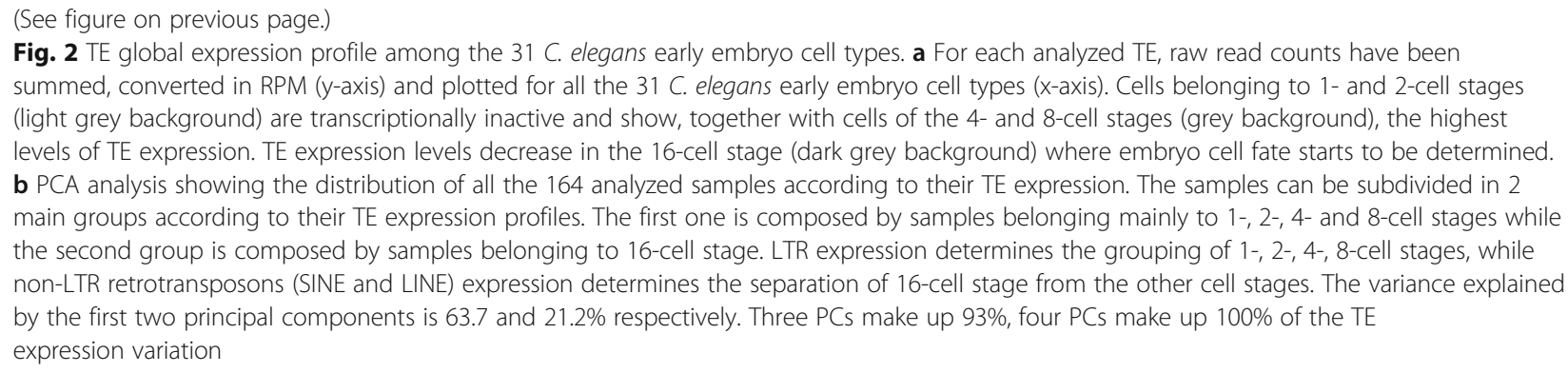

expression resulted low but detectable with a median number of TE-specific reads of $0.1 \%$ across all the samples. Interestingly, about $20 \%$ of reads mapping with at least one best alignment on TE belongs to the TE-non-specific reads. For these reads it is not possible to determine whether they originated from a coding/non-coding transcript or a TE and therefore, keeping them into account, might cause biased expression level calculations. We carried out the same expression analysis using SalmonTE [41], a recently published tool for TE expression. The results obtained with SalmonTE globally confirmed the general trends observed with our pipeline (Fig. 1c). However, especially in the AB descendant cells of the 16-cell stage, SalmonTE indicated generally higher TE expression levels with respect to our pipeline. To better understand the origin of the difference between the two sets of results, we selected all the TE-non-specific reads and quantified their level for each sample. The results (Fig. 1d) showed that TE-non-specific reads are more abundant in AB-descendant cells (16-cell stage), which correspond to the samples with the highest difference between SalmonTE and our pipeline. These results suggest that the differences observed between the two pipelines are mainly due to the different usage of TE-non-specific reads and that SalmonTE might be using, to measure TE expression levels, also reads which could be deriving from coding/non-coding transcripts. Intriguingly, $\mathrm{AB}$ cells of the 16-cell stage give also rise to neurons [34, 43, 44], which are known to be characterized by the expression of a high number of long noncoding RNAs (lncRNAs) which in turn are enriched for TE fragments [45-47]. We therefore believe that the usage of TE-non-specific reads in the quantification of TE expression might lead to an overestimation of TE expression, especially in nervous tissues, caused by the expression of annotated transcripts with embedded TE fragments. Filtering out TEnon-specific reads would lead to a more precise quantification of TE expression.

\section{TE expression changes among the stages of the $C$. elegans early embryo}

The TE global expression profiles in each of the 31 cell type and stage (raw read counts in Additional file 3) is summarized in Fig. 2a and in Additional file 4. It shows that TE abundance is particularly high in the transcriptionally inactive embryo cells (1-cell P0 zygote, 2-cell $\mathrm{AB}$ and $\mathrm{P} 1$ cells) [48], in the 4-cell stage and in the 8-cell stage. This suggests that TE mRNAs are a component of the maternal mRNAs and are important in the initial developmental stages. A principal component analysis performed on the expression levels of all the C. elegans TE belonging to DNA, LTR, LINE and SINE classes (Fig. 2b) shows that the 164 samples could be subdivided in two main groups. The first group mainly collects samples from the initial stages (1-, 2-, 4- and 8-cell stages), while the second group is principally composed by samples from the 16-cell stage. LTR expression determines the grouping of 1-, 2-, 4-, 8-cell stages, while non-LTR retrotransposons (SINE and LINE) expression determines the separation of 16-cell stage from the other cell stages, indicating that these two groups of elements have rather opposite expression dynamics. These results support the observation that LTR and non-LTR retrotransposon expression might be differentially regulated in the C. elegans early embryo.

\section{LTR expression is higher during stages associated to pluripotency maintenance and might activate the embryo innate immune response}

To better investigate TE classes expression patterns, we separated the TE according to the different classes and inspected their expression levels (Fig. 3). LTR retrotransposons (Fig. 3a) are the elements showing the highest expression levels in the C. elegans embryo. Overall, LTR are highly abundant in the initial stages of $C$. elegans embryo development (1-, 2-, 4- and 8-cell stages). In particular, LTR are highly expressed in the zygote (1-cell $\mathrm{P} 0$ ) and in almost all the AB cells of the 2-, 4- and 8-cell stages. Intriguingly, LTR expression decreases strongly in the 16-cell stage. CER1 and LTRCER1 are the two most expressed LTR elements (Additional files 4 and 5). Their expression profiles are very similar and recapitulate general LTR expression profile, since they are both highly expressed in 1-, 2-, 4- and 8-cell stage, while lowly expressed in 16-cell stage. Although the gastrulation process in C. elegans begins at the 26-cell stage [49], at the 16-cell stage the fate of all the embryo cells starts to be determined $[29,50]$ and consequently the number of pluripotent cells drops down. The deep decrease of LTR expression in correspondence 


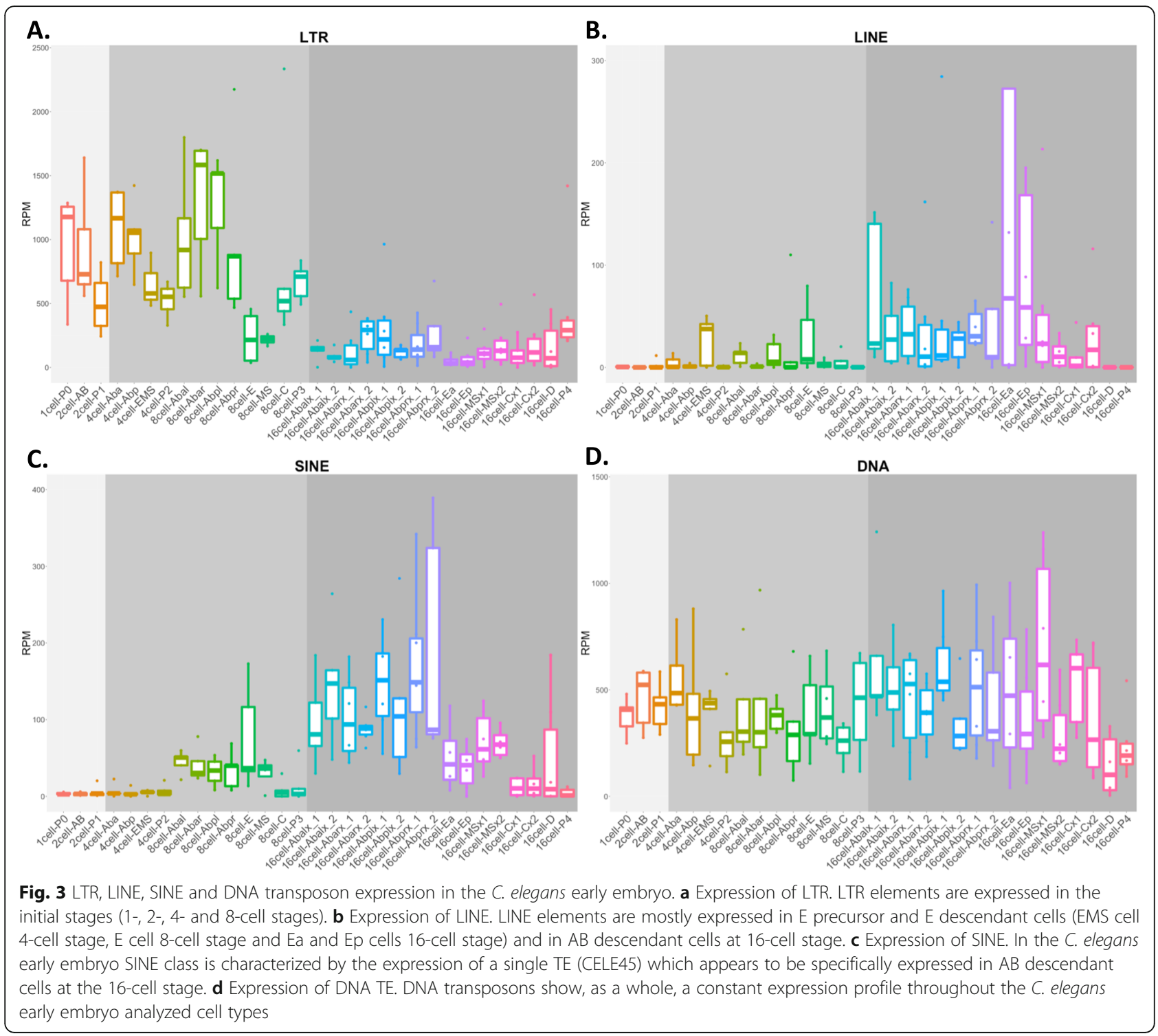

of the 16-cell stage may indicate that LTR are mostly expressed in undifferentiated cells, suggesting a role for LTR in the maintenance of pluripotency also in C. elegans as already reported in higher organisms. In mouse and human embryonic stem cells (ESCs), different classes of TE are specifically expressed across a transcriptional spectrum of pluripotency $[13,16]$. In addition, specific ERVs are reactivated during the reprogramming of somatic cells into induced pluripotent stem cells (iPSCs) [17]. In addition to pluripotency, it has also been shown that LTR-derived nucleic acids may play a role in the activation of innate immune pathways in mammals [51]. C. elegans lacks an adaptive immune system, however it does have an innate immune system and is able to respond to external insults from bacteria, fungi and viruses. The C. elegans innate immune system is composed by anti-viral and anti-microbial pathways: the anti-viral response is activated by viral double-strand RNA (dsRNA) and is mediated by the RNAi machinery, while the anti-microbial response is composed by different pathways whose induction led to the activation of secreted effector proteins such as C-type lectin anti-microbial peptides (AMPs) [52]. To our knowledge, LTR retrotransposons activity in relation to infections has not been studied in C. elegans. However, in higher organisms it has been suggested that LTR elements may have an immuno-protective role triggering the innate immune system and thus activating the embryo to respond to pathogens. Grow et al. studied, in ES cells, the activity of the LTR HERV-K, the most active human endogenous retrovirus family. HERV-K encodes a small accessory protein, Rec, homologous to HIV Rev., which allows nuclear export and translation of viral RNAs. In their work, Rec overexpression resulted in increased expression of viral restriction factors leading the authors to propose that 
HERV-K might provide an immunoprotective effect for human embryos, activating the innate immune system against different classes of viruses [14].

\section{LINE elements are mainly expressed in E lineage cells}

As shown in the Fig. 3b, LINE are the less expressed class of TE in the C. elegans early embryo. Overall, according to our analysis, LINE are expressed in few cell types, mainly belonging to the 16-cell stage. In particular, our results suggest that LINE are expressed in E and E precursor cells (4-cell stage EMS cell, 8-cell stage E cell and 16-cell stage Ea and Ep cells) and, at lower levels, in several AB cells of the 16cell stage. Intriguingly, the $\mathrm{E}$ lineage gives rise to the intestine $[34,53]$, while $A B$ lineage gives rise to neurons and non-neuronal tissues characterized by high concentration of nervous connections such as pharynx and epidermis [34, 43, $44,54,55]$. LINE expression in intestine precursor cells was quite unexpected, whereas the expression of LINE in neurons and nervous system associated tissues has already been observed for higher organism [5, 18-23] and will be discussed in the next paragraph. Our analyses evidenced that there is not a single element capable to recapitulate the LINE global expression pattern as resulted for LTR. The general expression profile observed is the sum of different elements showing variable and element-specific expression dynamics. LINE2A and LINE2C1 are mostly expressed in the 4-cell stage EMS cell and in MS cells (16-cell stage), LINE2B is expressed in the 8-cell stage E cell and in the 16cell stage $A B$ and MSx1 cells while LINE2F, that have an expression of $\sim 5$-fold with respect to LINE $2 \mathrm{~A}, 2 \mathrm{C} 1$ and $2 \mathrm{~B}$, seems to be exclusively expressed in Ea and Ep cells of the 16-cell stage (Additional files 4 and 6). This may suggest that different LINE elements might play different roles during $C$. elegans embryogenesis.

\section{SINE are mainly expressed in $A B$ lineage cells}

Figure 3c shows SINE element expression. SINE are expressed at higher levels with respect to LINE, but lower than LTR and DNA transposons. SINE class in the C. elegans reference genome is composed of 2 elements (SINE1 and CELE45), with CELE45 being the only one resulting expressed in our analysis (Additional file 4). CELE45 is highly expressed in all the $A B$ cells at the 16-cell stage, suggesting its specific expression in neurons, pharynx and epidermis precursors, as partially shown by LINE. The expression of CELE45 in AB cells at the 16-cell stage seems to be more specific than the expression of LINE and in particular of the LINE2B element. Taken together, our results suggest that both the SINE CELE45 and the LINE LINE2B are expressed in tissues characterized by associations to the nervous system. Expression and activity of non-LTR retrotransposons have already been evidenced in neurons and neuronal precursors in other species. Perrat et al. showed expression and insertional activity of several TE including
LINE-like elements in Drosophila melanogaster brains [5]. Moreover, several studies proposed that LINE elements are expressed and actively retrotransposed in neuronal precursors during differentiation of the central nervous system inducing somatic mosaicism and increasing the neuronal plasticity in mouse and human brains [18, 23]. Therefore, we speculate that activation of non-LTR elements in C. elegans nervous cells during development may play a role in neuronal cell fate specification, leading to neuronal cells diversity and possibly affecting neural plasticity and synapsis formation.

\section{DNA TE have a heterogeneous expression profiles}

DNA TE (Fig. 3d) are expressed at higher levels with respect to SINE and LINE but lower than LTR. DNA transposons are the most abundant TE in the C. elegans genome and they are the only class previously suggested to be active in the C. elegans genome [32, 33]. Their global expression is relatively constant throughout the analyzed stages and cell types. The most expressed DNA TE are Chapaev1, CEMUDR1, PALTA3, and PALTTTAAA3 (Additional files 4 and 7) and intriguingly these 4 TE have very different profile of expression. Chapaev1 is constantly expressed among the early embryo cell types and its expression recapitulates the overall expression of DNA transposons. CEMUDR1 is expressed in 1-, 2-, 4- and 8-cell stages, its expression profile is similar to the one showed by LTR elements. PALTA3 and PALTTTAAA3 elements are lowly expressed in 1-, 2- and 4-cell stages, their expression increases at 8-cell stage reaching the highest expression in the $A B$ cells of the 16-cell stage. This expression profile is very similar to the one showed by LINE2B and CELE45. These results suggest that DNA transposons have a heterogeneous expression profile that can be divided in the following types: i) constant, ii) LTR like and iii) non-LTR like. DNA transposons are therefore the only TE class constantly expressed in all the cell types of the C. elegans early embryo.

\section{Expression of LTR elements correlates with the expression of genes associated to the innate immune response} In the latest years, several studies reported that, particularly during the embryogenesis, TE may modulate gene expression [11-14]. For this reason, we carried out a correlation analysis between TE and gene expression profiles. Although this analysis does not specifically elucidate any direct interaction between TE and genes, it can highlight similarity in expression profiles that may suggest functional relationships. To perform this analysis, we took advantage of TE expression values calculated using our pipeline and gene expression values calculated in the work published by Tintori et al. [34]. To select TE and genes with reproducible expression levels among replicates of the different cell types we selected TE and genes with expression values higher than 25 in at least 3 replicates of at least 1 cell type. This 
led to the selection of $11 \mathrm{TE}$ and 6580 genes. We then performed the Pearson correlation test selecting all gene/TE pairs showing an expression correlation with $R^{2}>=0.4$ or $<$ $=-0.4$ and a corrected $p$-value $<0.0001$. This resulted in 1300 positively and 169 negatively correlated gene/TE pairs (Additional file 8). The 1300 positive correlations are determined by 1097 non redundant genes: 909 of these are correlated with 1 TE, 173 with 2 and 15 with 3 TE. The 169 negative correlations are determined by a set of 143 nonredundant genes, of which 117 are correlated with $1 \mathrm{TE}$ and 26 with 2 TE. The correlation analysis evidenced that the LTR elements CER1 and LTRCER1, the SINE CELE45 and the DNA CEMUDR determine the highest number of correlations, with CEMUR showing exclusively positive correlations, CELE45, CER1 and LTRCER1 both positive and negative ones (Table 1). We then performed an enrichment analysis to identify pathways associated to genes correlating with TE. This analysis evidenced 66 pathways significantly enriched in the groups of genes determining the identified correlations. Of these, 36 pathways result associated to genes positively correlated with 5 TE (CEMUDR, PALTTTAAA3, LINE2F, LTRCER1 and CER1) (Fig. 4a and Additional file 9) and 31 pathways are associated to genes negatively correlated with a single TE (CER1) (Fig. 4b and Additional file 10). The enriched pathways resulting by positive correlations can be classified in 7 main groups: DNA repair, immune system, metabolism, metabolism of proteins, metabolism of RNA, signal transduction, and vesicle-mediated transport. The enriched pathways resulting by genes negatively correlated with CER1 can be classified in 7 main groups: cell cycle, DNA replication, immune system, metabolism of proteins, metabolism of RNA, signal transduction, and transport of small molecules. Here, it is

Table 1 Number of positive and negative correlations for the 11 selected TEs

\begin{tabular}{lllll}
\hline TE & Class & Correlations & Positive corr. & Negative corr. \\
\hline CEMUDR1 & DNA & 202 & 202 & 0 \\
CEREP1A & DNA & 48 & 48 & 0 \\
Chapaev-1 & DNA & 4 & 4 & 0 \\
PALTA3 & DNA & 96 & 96 & 0 \\
PALTTTAAA3 & DNA & 104 & 104 & 0 \\
TC5 & DNA & 8 & 8 & 0 \\
LINE2F & LINE & 77 & 77 & 0 \\
CER1 & LTR & 363 & 323 & 40 \\
CER3-1 & LTR & 84 & 84 & 0 \\
LTRCER1 & LTR & 286 & 248 & 38 \\
CELE45 & SINE & 197 & 106 & 91 \\
\hline
\end{tabular}

1st column: list of the 11 TE with RPM $>25$ in at least 3 replicates of at least 1 cell type. 2nd column: TE classes (DNA, LINE, SINE, LTR). 3rd column: total number of correlations between TE and genes with RPKM $>25$ in at least 3 replicates of at least 1 cell type. 4th column: number of positive correlations. 5th column: number of negative correlations important to point out that, in some cases, pathway annotations for C. elegans might have been inferred by homology, transferring the annotations of homologous genes from more complex species. Results must be therefore interpreted with care considering the specific biological system under investigation. This is especially true for pleiotropic genes, with multiple functions, belonging to multiple pathways in complex organisms. The high number of functions for such genes is likely the result of their evolutionary recruitment into novel biological processes during the route leading to increased organismal complexity. For instance, our analysis identified a significant positive correlation between CER1 and innate immune system genes, a result in agreement with a possible involvement of CER1 in the embryonic activation of the innate immune response in C. elegans. On the other hand, this element also results negatively correlated with genes associated to the adaptive immune system, which is unlikely as C. elegans does not possess an adaptive immune response. However, these same genes are also annotated as belonging to the ubiquitination pathway, a function consistent with the biological system under analysis. Taking all this into account, we believe that our correlation analysis supports the conclusion that genes associated to the innate immune response are significantly enriched among those whose expression correlates with the expression of LTR elements, reinforcing our previous observations.

\section{Conclusions}

Several studies have recently reported the expression of TE in mammalian embryos and the CNS suggesting their role in fundamental biological processes such as pluripotency maintenance, embryo viability and differentiation, brain functioning, evolution and diversification [2, 12-14, 18-22]. In this study we developed a bioinformatics pipeline able to quantify reads specifically mapping on TE and explored TE expression in the C. elegans early embryo, from zygote to 16-cell stage. Our results suggest that, especially in neural tissues, a portion of reads mapping on TE cannot be distinguished by reads deriving from TE fragments embedded in annotated transcripts. These non-specific reads should therefore be discarded to avoid biases in the estimation of $\mathrm{TE}$ expression. In addition, our data show that TE are expressed in the $C$. elegans embryo and that, despite their low level of expression, they present different expression profiles in different embryonic stages and cell types, suggesting a specific regulation during early development. We observed a clear split of developmental TE expression levels in two phases characterized by the expression of two different families of TE, LTR and non-LTR. LTR elements resulted to be mostly expressed in the initial stages (1-, 2-, 4-, 8-cell stages). In particular, according to timing and territories of expression we propose that LTR expression (mainly LTRCER1 and CER1 elements) in the initial developmental 


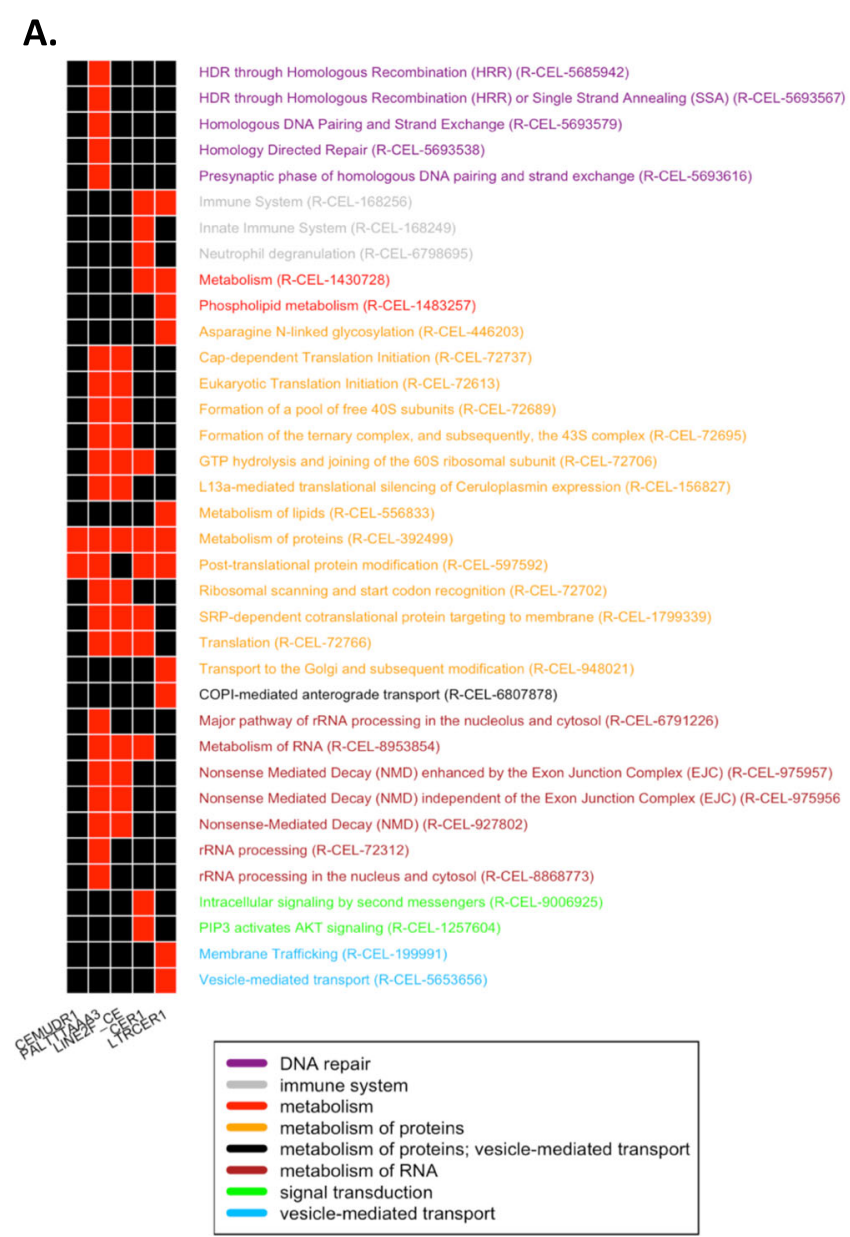

B.

Fig. 4 Pathways enriched in genes positively and negatively correlated with TEs. a Significantly enriched pathways associated to genes positively correlated with CEMUDR1, PALTTTAAA3, LINE2F, CER1 and LTRCER1. b Significantly enriched pathways associated to genes negatively correlated with CER1. (red color means presence, black absence)

stages might play a role in the maintenance of pluripotency and/or the innate immune response activation. We also observed that LINE are mostly expressed in intestine precursor cells (E lineage) and, together with CELE45 (SINE), in 16cell stage $A B$ cells, the ones giving rise to neurons and tissues connected with nervous system. These results are consistent with the observations reporting the expression of non-LTR elements in nervous tissues of other organisms like fruitfly, mouse and human [5, 18-23]. DNA transposons are the most abundant TE fixed in the C. elegans genome and, according to our results, the only TE class expressed in all the cell types of the C. elegans early embryo. Overall, DNA transposons are constantly expressed and are composed by TE with heterogeneous expression profiles that can be summarized in: i) constant (Chapaev1), ii) LTRlike (CEMUDR1) and iii) non-LTR-like (PALTA3 and PALTTTAAA3).

To our knowledge this is the first report analyzing expression of TE in the C. elegans early embryo and no work on the effects of TE silencing during the C. elegans development has ever been performed. In this work we have tried to support our speculations reasoning at a broader evolutionary context, taking into account experiments made in other organisms. Experiments of TE silencing in developmental and/or cellular contexts have been performed mainly in cultures of mammalian pluripotent stem cells and no comprehensive inspections on the effects of TE silencing during whole development of an entire embryo have been reported. In 2004 Park and colleagues [56] silenced, in the mouse zygote, the MT transposon like element, which belong to the LTR family and is expressed in the oocyte. The silencing resulted in the block of the zygote division thus suggesting a fundamental role played by a transposon during mouse embryogenesis. Lu et al. [57] silenced the LTR retrotransposon HERVH in hESC and observed a morphological change with cells adopting a fibroblast-like appearance. Furthermore, they also described a significant up-regulation of HERVH during the reprogramming of fibroblasts into induced pluripotent stem cells (iPSCs) supporting the involvement of the 
HERVH retrotransposon in the maintenance of the pluripotency state in hESCs. Future RNAi experiments of TE in $C$. elegans embryos might validate whether the expression of TE has any functional role. Departing from our results, a first indicative experiment would consist in silencing the most expressed LTR, LTRCER1 and CER1, followed by measuring the embryo susceptibility to viral and bacterial attacks and its capability to correctly develop and differentiate.

We propose that, despite the low level of expression, TE transcription is finely regulated during the early embryo development of $C$. elegans and might be involved in specific developmental functions in agreement and reinforcing what has already been observed in more complex organisms.

\section{Supplementary information}

Supplementary information accompanies this paper at https://doi.org/10. 1186/s12859-019-3088-7.

Additional file 1. TE occupancy in C. elegans, Drosophila, mouse and human reference genomes. Genomic occupancy of the 5 TE classes (DNA - light red, RC - ochre, LINE - light green, LTR - cyan and SINE - purple) in C. elegans, D. melanogaster, M. musculus and H. sapiens genomes. More than $70 \%$ of C. elegans TE are DNA transposons, $~ 60 \%$ of Drosophila TE are annotated as LTR and intriguingly Drosophila genome completely lacks SINE, $\sim 40 \%$ of mouse TE are annotated as SINE and $\sim 45 \%$ of human TE are LINE. TE annotation files for the 5 species have been retrieved from UCSC database (https://genome.ucsc.edu)

Additional file 2. SRA accession ID for the 164 analyzed samples. For each of the $31 \mathrm{C}$. elegans early embryo cell type is reported the NCBI-SRA database accession ID of the replicates that passed the quality filters regarding whole embryo mRNA mass according to the authors [34]. 1st column contains the names of the 31 cell types, the 2 nd contains the SRA ID of each replicate separated by commas.

Additional file 3. Raw read counts of the 163 C. elegans analyzed TE. Table containing the raw read counts measured with our pipeline. 1st column contains the names of the 163 analyzed TE. 2nd column contains the classes (DNA, LTR, LINE, RC, SINE) of the TE. Columns 3rd-166th contain the raw read counts for every sample measured with our pipeline.

Additional file 4. TE expression profiles in the C. elegans early embryo. For each TE belonging to DNA, LTR, LINE and SINE classes (rows) are reported the $\log _{10}(\mathrm{RPM})$ expression values of each replicate of the 31 cells analyzed (columns). Black color means no expression, green low and red high expression. The horizontal color band above the picture corresponds to different stages (1-, 2-, 4-, 8- and 16-cell stages - left to right), while the vertical color band on the left side of the picture indicates the TE classes (DNA, LTR, LINE, SINE - top to bottom).

Additional file 5. CER1 and LTRCER1 expression profiles in the C. elegans early embryo. A) CER1 and B) LTRCER1 expression profiles. CER1 and LTRCER1 are the most expressed LTR elements. The two LTR are expressed in the 1-, 2-, 4- and 8-cell stages while their expression in the 16 -cell stage is very low. Their expression profiles recapitulate the global expression pattern of LTR elements.

Additional file 6. Expression profiles of the 4 most expressed LINE. A) and C) LINE2A and LINE2C1 have similar expression profiles and are mostly expressed in EMS cell (4-cell stage) and in MSx2 cell (16-cell stage). B) LINE2B is expressed in 8-cell $E$, in $A B$ cells and in $M S \times 1$ cell of the 16-cell stage. D) LINE2F is expressed $\sim 5$-fold with respect to LINE $2 \mathrm{~A}$, $2 \mathrm{~B}$ and $2 \mathrm{C} 1$ and its expression seems to be related to 16-cell stage Ea and Ep cells.

Additional file 7. Expression profiles of the 4 most expressed DNA transposons. A) CEMUDR1 has an LTR-like expression profile: it is expressed in the 1-, 2-, 4- and 8-cell stages and not in the 16-cell stage.
B) Chapaev-1 has a constant expression profile that recapitulates the general DNA transposon class profile of expression. C) and D) PALTA3 and PALTTTAAA3 have a non-LTR-like profile of expression: these TE are mostly expressed in 16-cell stage AB cells.

Additional file 8. Significant correlations between TE and genes. Table containing the 1469 correlations with $R^{2}>=0.4$ or $<=-0.4$ and FDR corrected $p$-value $<0.0001$ between the 11 selected TE (expression value $>25$ RPM in in at least 3 replicates of at least 1 cell type) and the 6580 selected genes (expression value $>25$ RPKM in in at least 3 replicates of at least 1 cell type). 1st column contains TE names, 2 nd gene names, 3 rd $\mathrm{R}^{2}$ score, 4th $p$-value and 5th FDR corrected $p$-value.

Additional file 9. Enriched pathways associated to genes positively correlated with TE. 1st column contains significantly enriched Reactome pathways associated to genes positively correlated with $T E$, evidenced by Panther tool with an FDR corrected $p$-value $<0.01$. Columns 2 nd-6th contain absence (0) or presence (1) of the pathways in CEMUDR1, PALTTTAAA3, LINE2F, CER1, LTRCER1. 7th column contains FDR corrected $p$ value and 8 th the pathway classification.

Additional file 10. Enriched pathways associated to genes negatively correlated with TEs. 1st column contains over-represented Reactome pathways associated to genes negatively correlated with TE, evidenced by Panther tool with an FDR corrected $p$-value $<0.01$. Columns 2 nd -4 th contain absence (0) or presence (1) of the pathways in CELE45, CER1 and LTRCER1. 5th column contains FDR corrected $p$-value and 6th the pathway classification.

\section{Abbreviations}

C. elegans: Caenorhabditis elegans; CNS: Central nervous system;

ESCs: Embryonic stem cells; iPSCs: Induced pluripotent stem cells; LINE: Long interspersed nuclear elements; IncRNAs: Long non-coding RNAs; LTR: Long terminal repeats; RC: Rolling-circle; RNAi: LTR RNA interference; RPM: Reads per million mapped reads; sc-RNAseq: Single-cell RNA-seq; SINE: Short interspersed nuclear elements; TE: Transposable elements

\section{Acknowledgements}

The authors would like to thank the guest editors and the anonymous reviewers. Furthermore, the authors would like to thank Dr. Greta Busseni (Integrative Marine Ecology - Stazione Zoologica Anton Dohrn, Napoli, Italy) for her support and the authors of the Tintori et al. article and members of the Goldstein lab, without their data made public this study could have not been possible.

\section{About this supplement}

This article has been published as part of BMC Bioinformatics, Volume 20 Supplement 9, 2019: Italian Society of Bioinformatics (BITS): Annual Meeting 2018. The full contents of the supplement are available at https:// bmcbioinformatics.biomedcentral.com/articles/supplements/volume-20supplement-9.

\section{Authors' contributions}

RS, FA, SG designed the study; FA performed all analyses and wrote the manuscript; RS supervised the analyses, wrote and reviewed the manuscript; SG, MS and EDS reviewed the manuscript; all the authors approved the final version of the manuscript.

Funding

Publication costs are funded by Istituto Italiano di Tecnologia (IIT).

\section{Availability of data and materials}

The datasets analyzed during the current study are available in the ENA EBI repository (PRJNA312176) https://www.ebi.ac.uk/ena/data/view/PRJNA312176

Ethics approval and consent to participate

Not applicable

Consent for publication

Not applicable 


\section{Competing interests}

The authors declare that they have no competing interests.

\section{Author details}

${ }^{1}$ Area of Neuroscience, International School for Advanced Studies (SISSA), Trieste, Italy. ${ }^{2}$ Central RNA Laboratory, Istituto Italiano di Tecnologia (IIT), Genova, Italy. ${ }^{3}$ Institute of Biosciences and BioResources (IBBR), CNR, Napoli, Italy. ${ }^{4}$ Biology and Evolution of Marine Organisms, Stazione Zoologica Anton Dohrn, Napoli, Italy.

\section{Received: 22 August 2019 Accepted: 10 September 2019} Published: 22 November 2019

\section{References}

1. Wicker T, Sabot F, Hua-Van A, Bennetzen JL, Capy P, Chalhoub B, et al. A unified classification system for eukaryotic transposable elements. Nat Rev Genet. 2007;8:973-82.

2. Feschotte $\mathrm{C}$. Transposable elements and the evolution of regulatory networks. Nat Rev Genet. 2008;9:397-405.

3. The FANTOM Consortium. The transcriptional landscape of the mammalian genome. Science. 2005;309:1559-63.

4. Johnson R, Guigo R. The RIDL hypothesis: transposable elements as functional domains of long noncoding RNAs. RNA. 2014;20:959-76.

5. Perrat PN, DasGupta S, Wang J, Theurkauf W, Weng Z, Rosbash M, et al. Transposition-driven genomic heterogeneity in the Drosophila brain. Science. 2013;340:91-5.

6. Piacentini L, Fanti L, Specchia V, Bozzetti MP, Berloco M, Palumbo G, et al. Transposons, environmental changes, and heritable induced phenotypic variability. Chromosoma. 2014;123:345-54.

7. Engels WR. The P family of transposable elements in Drosophila. Annu Rev Genet. 1983:17:315-44

8. Tabara H, Sarkissian M, Kelly WG, Fleenor J, Grishok A, Timmons L, et al. The rde-1 gene, RNA interference, and transposon silencing in C. elegans. Cell. 1999;99:123-32.

9. Aravin AA, Hannon GJ, Brennecke J. The Piwi-piRNA pathway provides an adaptive defense in the transposon arms race. Science. 2007;318:761-4.

10. Kunarso G, Chia N-Y, Jeyakani J, Hwang C, Lu X, Chan Y-S, et al. Transposable elements have rewired the core regulatory network of human embryonic stem cells. Nat Genet. 2010:42:631-4.

11. Rodriguez-Terrones $D$, Torres-Padilla M-E. Nimble and ready to mingle: transposon outbursts of early development. Trends Genet. 2018;34(10):806-20.

12. Garcia-Perez JL, Widmann TJ, Adams IR. The impact of transposable elements on mammalian development. Development. 2016;143:4101-14.

13. Hackett JA, Kobayashi T, Dietmann S, Surani MA. Activation of lineage regulators and transposable elements across a pluripotent spectrum. Stem Cell Reports. 2017:8:1645-58.

14. Grow EJ, Flynn RA, Chavez SL, Bayless NL, Wossidlo M, Wesche DJ, et al. Intrinsic retroviral reactivation in human preimplantation embryos and pluripotent cells. Nature. 2015;522:221-5.

15. Percharde M, Lin C-J, Yin Y, Guan J, Peixoto GA, Bulut-Karslioglu A, et al. A LINE1-Nucleolin partnership regulates early development and ESC identity. Cell. 2018;174:391-405.e19.

16. Göke J, Lu X, Chan Y-S, Ng H-H, Ly L-H, Sachs F, et al. Dynamic transcription of distinct classes of endogenous retroviral elements marks specific populations of early human embryonic cells. Cell Stem Cell. 2015;16:135-41.

17. Friedli M, Turelli P, Kapopoulou A, Rauwel B, Castro-Díaz N, Rowe HM, et al. Loss of transcriptional control over endogenous retroelements during reprogramming to pluripotency. Genome Res. 2014;24:1251-9.

18. Coufal NG, Garcia-Perez JL, Peng GE, Yeo GW, Mu Y, Lovci MT, et al. L1 retrotransposition in human neural progenitor cells. Nature. 2009:460:1127-31.

19. Erwin JA, Paquola ACM, Singer T, Gallina I, Novotny M, Quayle C, et al. L1associated genomic regions are deleted in somatic cells of the healthy human brain. Nat Neurosci. 2016:19:1583-91.

20. Erwin JA, Marchetto MC, Gage FH. Mobile DNA elements in the generation of diversity and complexity in the brain. Nat Rev Neurosci. 2014;15:497-506.

21. Richardson SR, Morell S, Faulkner GJ. L1 retrotransposons and somatic mosaicism in the brain. Annu Rev Genet. 2014:48:1-27.

22. Baillie JK, Barnett MW, Upton KR, Gerhardt DJ, Richmond TA, De Sapio F, et al. Somatic retrotransposition alters the genetic landscape of the human brain. Nature. 2011;479:534-7.
23. Muotri AR, Chu VT, Marchetto MCN, Deng W, Moran JV, Gage FH. Somatic mosaicism in neuronal precursor cells mediated by L1 retrotransposition. Nature. 2005:435:903-10

24. Krug L, Chatterjee N, Borges-Monroy R, Hearn S, Liao W-W, Morrill K, et al. Retrotransposon activation contributes to neurodegeneration in a Drosophila TDP-43 model of ALS. PLoS Genet. 2017;13:e1006635 Feschotte C, editor.

25. Guo C, Jeong H-H, Hsieh Y-C, Klein H-U, Bennett DA, De Jager PL, et al. Tau activates transposable elements in Alzheimer's disease. Cell Rep. 2018;23:2874-80.

26. Tan H, Wu C, Jin L. A possible role for long interspersed nuclear elements-1 (LINE1) in Huntington's disease progression. Med Sci Monit. 2018;24:3644-52.

27. Sun W, Samimi H, Gamez M, Zare H, Frost B. Pathogenic tau-induced piRNA depletion promotes neuronal death through transposable element dysregulation in neurodegenerative tauopathies. Nat Neurosci. 2018:21:1038-48.

28. Corsi AK, Wightman B, Chalfie M. A Transparent Window into Biology: A Primer on Caenorhabditis elegans. Genetics. 2015:200:387-407.

29. Sulston JE, Schierenberg E, White JG, Thomson JN. The embryonic cell lineage of the nematode Caenorhabditis elegans. Dev Biol. 1983;100:64-119.

30. Fire A, Xu S, Montgomery MK, Kostas SA, Driver SE, Mello CC. Potent and specific genetic interference by double-stranded RNA in Caenorhabditis elegans. Nature. 1998;391:806-11.

31. Grishok A, Mello CC. RNAi (nematodes: Caenorhabditis elegans). Adv Genet. 2002:46:339-60.

32. Laricchia KM, Zdraljevic S, Cook DE, Andersen EC. Natural variation in the distribution and abundance of transposable elements across the Caenorhabditis elegans species. Mol Biol Evol. 2017;34:2187-202.

33. Bessereau J-L. Transposons in C. elegans. WormBook. 2006; Available from: http://www.wormbook.org/chapters/www_transposons/transposons.html. Cited 27 Mar 2018.

34. Tintori SC, Osborne Nishimura E, Golden P, Lieb JD, Goldstein B. A transcriptional lineage of the Early C. elegans embryo. Dev Cell. 2016:38:430-44.

35. Zerbino DR, Achuthan P, Akanni W, Amode MR, Barrell D, Bhai J, et al Ensembl 2018. Nucleic Acids Res. 2018:46:D754-61.

36. Bao W, Kojima KK, Kohany O. Repbase update, a database of repetitive elements in eukaryotic genomes. Mob DNA. 2015;6:11.

37. Dobin A, Davis CA, Schlesinger F, Drenkow J, Zaleski C, Jha S, et al. STAR: ultrafast universal RNA-seq aligner. Bioinformatics. 2013;29:15-21.

38. Li H, Handsaker B, Wysoker A, Fennell T, Ruan J, Homer N, et al. The sequence alignment/map format and SAMtools. Bioinformatics. 2009:25:2078-9.

39. Picard tool - Broad Institute. Available from: http://broadinstitute.github.io/ picard/. Cited 10 Mar 2018.

40. Quinlan AR, Hall IM. BEDTools: a flexible suite of utilities for comparing genomic features. Bioinformatics. 2010;26:841-2.

41. Jeong H-H, Yalamanchili HK, Guo C, Shulman JM, Liu Z. An ultra-fast and scalable quantification pipeline for transposable elements from next generation sequencing data. Pac Symp Biocomput. 2018;23:168-79.

42. Thomas PD, Campbell MJ, Kejariwal A, Mi H, Karlak B, Daverman R, et al. PANTHER: a library of protein families and subfamilies indexed by function. Genome Res. 2003;13:2129-41.

43. Altun ZF, Hall DH. Nervous system, general description. WormBook. 2011; Available from: http://www.wormatlas.org/hermaphrodite/nervous/ Neuroframeset.html. Cited 28 Mar 2018.

44. Hobert O. Neurogenesis in the nematode Caenorhabditis elegans. WormBook. 2010; Available from: http://www.wormbook.org/chapters/ www_specnervsys.2/neurogenesis.html. Cited 27 Mar 2018.

45. Carrieri C, Cimatti L, Biagioli M, Beugnet A, Zucchelli S, Fedele S, et al. Long non-coding antisense RNA controls Uchl1 translation through an embedded SINEB2 repeat. Nature. 2012;491:454-7.

46. Nishihara H, Smit AFA, Okada N. Functional noncoding sequences derived from SINEs in the mammalian genome. Genome Res. 2006;16:864-74.

47. Ponicsan SL, Kugel JF, Goodrich JA. Genomic gems: SINE RNAs regulate mRNA production. Curr Opin Genet Dev. 2010;20:149-55.

48. Osborne Nishimura E, Zhang JC, Werts AD, Goldstein B, Lieb JD. Asymmetric transcript discovery by RNA-seq in C. elegans blastomeres identifies neg-1, a gene important for anterior morphogenesis. PLoS Genet. 2015;11:e1005117.

49. Nance J, Lee J-Y, Goldstein B. Gastrulation in C. elegans. WormBook. 2005; Available from: http://www.wormbook.org/chapters/www gastrulation/ gastrulation.html. Cited 11 Sept 2018

50. Maduro MF. Cell fate specification in the C elegans embryo. Dev Dyn. 2010:1315-29.

51. Kassiotis G, Stoye JP. Immune responses to endogenous retroelements: taking the bad with the good. Nat Rev Immunol. 2016;16:207-19. 
52. Ermolaeva MA, Schumacher B. Insights from the worm: the C. elegans model for innate immunity. Semin Immunol. 2014;26:303-9.

53. McGhee J. The C. elegans intestine. WormBook. 2007; Available from: http://www. wormbook.org/chapters/www intestine/intestine.html. Cited 5 July 2018.

54. Chisholm AD, Xu S. The Caenorhabditis elegans epidermis as a model skin. II: differentiation and physiological roles. Wiley Interdiscip Rev Dev Biol. 2012;1:879-902.

55. Altun ZF, Hall DH. Alimentary System, Pharynx. WormBook. 2009; Available from: http://www.wormatlas.org/hermaphrodite/pharynx/Phaframeset.html. Cited 28 Mar 2018.

56. Park C-E, Shin M-R, Jeon E-H, Lee S-H, Cha K-Y, Kim K, et al. Oocyte-selective expression of MT transposon-like element, clone MTi7 and its role in oocyte maturation and embryo development. Mol Reprod Dev. 2004;69:365-74.

57. Lu X, Sachs F, Ramsay L, Jacques P-É, Göke J, Bourque G, et al. The retrovirus HERVH is a long noncoding RNA required for human embryonic stem cell identity. Nat Struct Mol Biol. 2014;21:423-5.

\section{Publisher's Note}

Springer Nature remains neutral with regard to jurisdictional claims in published maps and institutional affiliations.

Ready to submit your research? Choose BMC and benefit from:

- fast, convenient online submission

- thorough peer review by experienced researchers in your field

- rapid publication on acceptance

- support for research data, including large and complex data types

- gold Open Access which fosters wider collaboration and increased citations

- maximum visibility for your research: over $100 \mathrm{M}$ website views per year

At BMC, research is always in progress.

Learn more biomedcentral.com/submissions 